\title{
CANONICAL MODELS AND THE LAW OF REQUISITE VARIETY
}

John L. Casti

International Institute for Applied Systems Analysis,

Laxenburg, Austria

RR-86-1

March 1986

Reprinted from Journal of Optimization Theory and Applications, volume 46 (1985).

INTERNATIONAL INSTITUTE FOR APPLIED SYSTEMS ANALYSIS

Laxenburg, Austria 
Research Reports, which record research conducted at IIASA, are independently reviewed before publication. However, the views and opinions they express are not necessarily those of the Institute or the National Member Organizations that support it.

Reprinted with permission from Journal of Optimization Theory and Applications, 46 (1985), 455-459.

Copyright @ 1985 Plenum Publishing Corporation (New York).

All rights reserved. No part of this publication may be reproduced or transmitted in any form or by any means, electronic or mechanical, including photocopy, recording, or any information storage or retrieval system, without permission in writing from the copyright holder.

Printed by Novographic, Vienna, Austria 


\section{FOREWORD}

One of the goals of the Science and Technology Program at the International Institute for Applied Systems Analysis is to develop theoretical modeling frameworks suitable for characterizing processes in business and industry. Two of many possible modeling frameworks derive from the cybernetic theory of information processing systems and the theory of linear control systems. In this paper, John Casti demonstrates that the main theoretical foundations of Ashby's theory of cybernetic processes, the so-called law of requisite variety and the fundamental theorem of linear realization theory, are equivalent. This result enables a bridge to be made between the general laws of cybernetic processes and the specific results pertaining to linear systems.

BORIS SEGERSTAHL

Leader

Science and Technology Program 


\title{
Canonical Models and the Law of Requisite Variety ${ }^{1}$
}

\author{
J. L. CASTI ${ }^{2}$
}

\author{
Dedicated to G. Leitmann
}

\begin{abstract}
The law of requisite variety from cybernetics is shown to be related to the reachability and observability properties of a dynamical control system. In particular, it is established that the transmission of all input variety to the system output is possible if and only if the system is canonical, i.e., completely reachable and completely observable.
\end{abstract}

Key Words. Requisite variety, canonical systems, reachability, observability.

\section{Cybernetics and the Law of Requisite Variety}

In the thoery of cybernetic control, as described for instance in the classical work of Ashby (Ref. 1), a central role is played by the concept of the variety of a regulator $R$. Roughly speaking, the variety of $R$ is the number of distinct inputs that the regulator can apply to the system during the course of its operation (in cybernetics, it is usually taken as the logarithm of this number). Ashby showed that the ability of any regulator to control a given system is severely constrained by a control-theoretic version of the second law of thermodynamics, the so-called law of requisite variety.

There are many equivalent statements of this pivotal result, the one most appropriate for our purposes being: "the capacity of $R$ as a regulator cannot exceed $R$ 's capacity as a channel of communication".

In control terms, the above law of requisite variety means that, in order for all of the variety present in $R$ to be transmitted to the system, the communication channel linking $R$ to the system must be capable of transmitting the full variety of $R$. When stated in such terms, the law seems obvious and trivial; the objective of this note is to show that it is not.

\footnotetext{
'This work was partially supported by the National Science Foundation under Grants Nos. CEE-81-00491 and CEE-81-10778.

${ }^{2}$ International Institute for Applied Systems Analysis, Laxenburg, Austria.
} 
To classical engineering-oriented control theorists, there is something annoyingly elusive about the law of requisite variety, at least in its above form. On the one hand, it is clearly a statement about the regulation of a dynamical system, the bread-and-butter of a control theorist's daily life. In fact, it is an extremely broad statement making no distinction about the nature of the regulator $R$, the system, or the channel linking them. On the other hand, it seems less than straightforward to see how to fit this general principle into the usual $\dot{x}=f(x, u)$ framework that is the mathematical starting point for most control theoretic investigations. The only work in this direction that we are aware of is a paper by Porter (Ref. 2), which basically just points out that there are many important control problems that are not easily accommodated by the standard framework, and that a more thorough examination of Ashby's ideas would likely bear fruit in this regard.

This paper represents a first step in bridging the gap between control theory and cybernetics by showing the relationship between the law of requisite variety and the idea of a canonical dynamical system, i.e., a system that is completely reachable and completely observable (Refs. 3-5). For illustrative purposes and to conserve space, we prove the following result for single-input/single-output linear systems.

Theorem 1.1. The variety in a system's input equals the variety in its output if and only if the system is canonical.

In view of the generality of Ashby's results, together with recent developments in nonlinear system theory (Refs. 6-8), there is little reason to doubt that our main theorem extends to more general situations. We shall examine some aspects of this extension at the end of the paper.

\section{Linear Systems and Variety}

Consider the discrete-time, single-input/single-output, constant linear system $\Sigma$,

$$
\begin{aligned}
x_{t+1} & =F x_{t}+g u_{t}, \quad x_{0}=0, \\
y_{t} & =h^{\prime} x_{t} .
\end{aligned}
$$

To avoid unnecessary technical complications, assume that $x \in k^{n}$, where $k$ is a finite field. Thus, $F$ is an $n \times n$ matrix over $k$, while $g, h \in k^{n}$.

Standard results from linear system theory show that $\sum$ is:

(A) completely reachable if, and only if the vectors $\left\{g, F g, F^{2} g, \ldots, F^{n-1} g\right\}$ are linearly independent; 
(B) completely observable if and only if the vectors $\left\{h^{\prime}, h^{\prime} F^{\prime}\right.$, $\left.h^{\prime}\left(F^{\prime}\right)^{2}, \ldots, h^{\prime}\left(F^{\prime}\right)^{n-1}\right\}$ are linearly independent.

In linear system theory, a reachable and observable system $\sum$ is termed canonical.

Consider an input sequence $U=\left\{u_{0}, u_{1}, \ldots, u_{n-1}\right\}$. If, at each moment $i$, the input $u_{i}$ can assume any of $M$ values from $k$, then we define the input variety to be $M^{n}$. By virtue of our assumption that $k$ is a finite field, the input variety is also finite. Clearly, the input variety is equals the number of distinguishable input sequences $U$ over an $n$-step time horizon. We define the state variety and output variety in a similar fashion to be the number of distinguishable state and output sequences, respectively. We can now state the following result.

Theorem 2.1. The input variety of $\sum$ equals the state variety if and only if $\sum$ is completely reachable.

Proof. At time $t=i$, the state of $\sum$ is given by

$$
x_{i}=\sum_{j=1}^{i} F^{j-1} g u_{i-j}, \quad i=1,2, \ldots, n .
$$

Assume that $\sum$ is completely reachable. Then, the elements $\left\{g, F g, \ldots, F^{n-1} g\right\}$ are linearly independent, which means that for each distinguishable input sequence $U=\left\{u_{0}, u_{1}, \ldots, u_{n-1}\right\}$, there is a corresponding distinguishable state sequence $X=\left\{x_{1}, x_{2}, \ldots, x_{n}\right\}$; i.e., the input variety equals the state variety.

Now, let the input variety equal the state variety; i.e., no two input sequences give rise to the same state sequence. But, by the above representation for the state, each state is a triangular linear combination of the elements $\left\{g, F g, \ldots, F^{n-1} g\right\}$. Thus, if each distinguishable input sequence gives rise to a different state sequence, the elements $\left\{g, F g, \ldots, F^{n-1} g\right\}$ must be linearly independent, i.e., $\sum$ is completely reachable.

Corollary 2.1. The state variety of $\sum$ equals its output variety if and only if $\sum$ is completely observable.

Proof. The proof is by means of duality, letting $g \rightarrow h^{\prime}, F \rightarrow F^{\prime}$.

Putting Theorem 2.1 together with Corollary 2.1, we obtain the main theorem stated earlier.

The above result can be interpreted more in line with the cybernetic law of requisite variety, if we regard the state space of $\sum$ as a channel by 
which the system inputs influence the outputs. The main theorem then states that the variety present in the regulator can be completely used to regulate the system's behavioral output if and only if $\sum$ is canonical.

\section{Discussion}

The law of requisite variety is a general statement about any control system; our main theorem is a very special result about a discrete-time, linear, single-input/single-output system over a finite field. Let us consider how our special assumptions can be removed in order to close this gap.

(i) Finite Field. The proof of Theorem 2.1 made no essential use of the fact that $k$ is a finite field. We only require that the notion of linear independence be well defined; hence, $k$ could be taken to be any integral domain, for instance. Thus, the finiteness assumption can be completely removed.

(ii) Single-Input/Single-Output. If $u \in k^{m}, y \in k^{p}, m, p>1$, there is also no problem, as we require only that the input and output varieties be no greater than that which the state is capable of displaying. Consequently, as long as we impose the bound $m, p \leqslant n$, the proof of Theorem 2.1 can be "souped-up" to accommodate multiple-input/multiple-output systems. Again, the essential factor is that different input sequences give rise to different state sequences which, in turn, generate different output sequences. This property is exactly what characterizes a canonical system.

(iii) Discrete Time. All reachability/observability results apply to continuous-time systems, as well as to discrete-time systems with minor modifications. The characterization of reachability/observability that we have used, involving the linear independence of $\left\{g, F g, \ldots, F^{n-1} g\right\}$, is independent of the time set employed.

(iv) Linearity. The underlying notion of complete reachability/observability as being characterized by distinguishable input/output sequences giving rise to distinguishable state sequences has been employed to define canonical systems, even for nonlinear dynamics. However, the linear independence arguments and algebraic criteria used in our proof have been extended to the nonlinear case only to show some form of local reachability/observability (Refs. 6-8). For special classes of nonlinearities (e.g., bilinear, polynomial) a global extension is possible, but, in general only local results are available. 
In conclusion, we see that the law of requisite variety is, in systemtheoretic terms, a statement about the way in which system states can be affected by inputs and how they can generate outputs. Just like its physics counterpart, the second law of thermodynamics, the law of requisite variety imposes an upper bound on the information that can be transmitted from a sender (the input) to a receiver (the output), with the maximum transfer being achieved by a canonical system. Thus, in some sense, we can say that a completely reachable and observable system is analogous to a noiseless communication channel.

\section{References}

1. Ash BY, W., An Introduction to Cybernetics, Chapman and Hall, London, England, 1956.

2. PORTER, B., Requisite Variety in Systems and Control Sciences, International Journal on Genetic Systems, Vol. 2, pp. 225-229, 1976.

3. CASTI, J., Dynamical Systems and Their Application: Linear Theory, Academic Press, New York, New York, 1977.

4. Brockett, R., Finite-Dimensional Linear Systems, John Wiley and Sons, New York, New York, 1970.

5. ZADEH, L., and Desoer, C. A., Linear Systems Theory, McGraw-Hill, New York, New York, 1963.

6. HermANN, R., and KRENER, A., Nonlinear Controllability and Observability, IEEE Transactions on Automatic Control, Vol. AC-22, pp. 728-740, 1977.

7. CASTI, J., Recent Developments and Future Perspectives in Nonlinear System Theory, SIAM Review, Vol. 24, pp. 301-331, 1982.

8. CASti, J., Nonlinear System Theory, Academic Press, New York, New York, 1985. 
